\title{
The predictive role of preoperative serum glutamate dehydrogenase levels in microvascular invasion and hepatocellular carcinoma prognosis following liver transplantation-a single center retrospective study
}

\author{
Jinlong Gong Equal first author, 1 , Yaxiong $\mathrm{Li}^{\text {Equal first author, } 2}{ }^{2}, \mathrm{Jia} \mathrm{Yu}^{1}$, Tielong Wang ${ }^{1}$, Jinliang Duan ${ }^{1}$, Anbin $\mathrm{Hu}^{1}$, Xiaoshun $\mathrm{He}^{1}$ \\ , Xiaofeng Zhu ${ }^{\text {Corresp. } 1}$ \\ ${ }^{1}$ Department of Organ Transplantation, The First Affiliated Hospital, Sun Yat-Sen University, Guangzhou, Guangdong province, China \\ 2 Department of Pancreato-Biliary Surgery, The First Affiliated Hospital, Sun Yat-Sen University, Guangzhou, Guangdong province, China \\ Corresponding Author: Xiaofeng Zhu \\ Email address: zhuxiaof@mail.sysu.edu.cn
}

Background: As a critical metabolic substrate, glutamine is not only involved in the progression of many cancers but is also related to angiogenesis. Glutamate dehydrogenase (GLDH), a key enzyme in glutamine metabolism, has been reported to regulate tumor proliferation; however, its relationship with microvascular invasion (MVI) is unclear. This study evaluated the ability of preoperative serum GLDH levels to predict MVI and the long-term survival of hepatocellular carcinoma (HCC) patients after liver transplantation (LT). Methods: HCC patients that underwent LT from January 2015 to May 2020 at the First Affiliated Hospital of Sun Yat-Sen University were enrolled in our retrospective analysis. Clinicopathological variables were extracted from medical records. A receiver operating characteristic curve was created to determine the optimal cut-off value of GLDH for MVI. Results: Preoperative GLDH was significantly elevated in the MVIpositive group $(U=454.00, p=0.000)$. The optimal cut-off value of GLDH for MVI was 7.45 $\mathrm{U} / \mathrm{L}$, with an area under the curve of 0.747 (95\% confidence interval: $0.639-0.856, p=$ 0.000 ). The sensitivity was $79.3 \%$, while the specificity was $64.5 \%$. GLDH $>7.45 \mathrm{U} / \mathrm{L}(p=$ $0.023)$ and maximum diameter $>5 \mathrm{~cm}(p=0.001)$ were independent risk factors for the presence of MVI. Patients with GLDH $>7.45 \mathrm{U} / \mathrm{L}$ had significantly poorer overall survival ( $p$ $=0.001)$ and recurrence-free survival $(p=0.001)$ after LT than patients with GLDH $\leq 7.45$ $\mathrm{U} / \mathrm{L}$. Similarly, patients with MVI were associated with poor survival $(p=0.000)$.

Conclusions: Preoperative elevated serum GLDH levels predict MVI and poorer long-term survival for HCC after LT. 
1 The predictive role of preoperative serum glutamate dehydrogenase levels in microvascular

2 invasion and hepatocellular carcinoma prognosis following liver transplantation-a single

3 center retrospective study

4

5 Jinlong Gong ${ }^{1 \#}$, Yaxiong $\mathrm{Li}^{2}{ }^{2 \#}$, Jia $\mathrm{Yu}^{1}$, Tielong Wang ${ }^{1}$, Jinliang Duan ${ }^{1}$, Anbin $\mathrm{Hu}^{1}$, Xiaoshun

$6 \mathrm{He}^{1}$, Xiaofeng $\mathrm{Zhu}^{1}$

8 'Department of Organ Transplantation, The First Affiliated Hospital, Sun Yat-Sen University,

9 Guangzhou, Guangdong province, China

10 2Department of Pancreato-Biliary Surgery, The First Affiliated Hospital, Sun Yat-Sen

11 University, Guangzhou, Guangdong province, China

Corresponding Author:

14 Xiaofeng Zhu ${ }^{1}$

15 Zhongshan Er road NO.58, Guangzhou, 510080, China

\#These authors contributed equally to this work. 


\section{Abstract}

Background: As a critical metabolic substrate, glutamine is not only involved in the progression of many cancers but is also related to angiogenesis. Glutamate dehydrogenase (GLDH), a key enzyme in glutamine metabolism, has been reported to regulate tumor proliferation; however, its relationship with microvascular invasion (MVI) is unclear. This study evaluated the ability of preoperative serum GLDH levels to predict MVI and the long-term survival of hepatocellular carcinoma (HCC) patients after liver transplantation (LT).

Methods: HCC patients that underwent LT from January 2015 to May 2020 at the First Affiliated Hospital of Sun Yat-Sen University were enrolled in our retrospective analysis. Clinicopathological variables were extracted from medical records. A receiver operating characteristic curve was created to determine the optimal cut-off value of GLDH for MVI.

Results: Preoperative GLDH was significantly elevated in the MVI-positive group $(U=454.00$, $p=0.000$ ). The optimal cut-off value of GLDH for MVI was $7.45 \mathrm{U} / \mathrm{L}$, with an area under the curve of 0.747 (95\% confidence interval: $0.639-0.856, p=0.000)$. The sensitivity was $79.3 \%$, while the specificity was $64.5 \%$. GLDH $>7.45 \mathrm{U} / \mathrm{L}(p=0.023)$ and maximum diameter $>5 \mathrm{~cm}$ $(p=0.001)$ were independent risk factors for the presence of MVI. Patients with GLDH $>7.45$ $\mathrm{U} / \mathrm{L}$ had significantly poorer overall survival $(p=0.001)$ and recurrence-free survival $(p=0.001)$ after LT than patients with GLDH $\leq 7.45 \mathrm{U} / \mathrm{L}$. Similarly, patients with MVI were associated with poor survival $(p=0.000)$.

Conclusions: Preoperative elevated serum GLDH levels predict MVI and poorer long-term survival for HCC after LT.

\section{Introduction}

Hepatocellular carcinoma (HCC) accounts for $85-90 \%$ of all primary liver cancer cases (Zhou et al., 2018). It is the fourth most common malignancy and the third leading cause of tumor-related deaths in China (Chen et al., 2016). Liver transplantation (LT) is considered the definitive treatment option for HCC as it removes not only the tumor but also the cirrhotic liver, which has the potential to develop new HCC lesions (Yang et al., 2019). However, recurrence post-LT is a major problem that threatens the long-term survival of HCC patients. Currently, even if the Milan or University of California San Francisco (UCSF) criteria are used to select transplant recipients, the probability of HCC recurrence post-LT ranges from $16 \%$ to $33 \%$ (Verna et al., 2020; Xu et al., 2016), with the median survival post-recurrence being only 10.612.2 months (Bodzin et al., 2017; Sapisochin et al., 2015). These numbers indicate that the traditional recipient selection and organ allocation criteria, which are based on tumor burden, do not always reflect tumor biology. Alternatively, molecular biomarkers may be superior in revealing tumor aggressiveness (Felden \& Villanueva, 2020).

Given the scarcity of available organs for transplantation, the recurrence risk in HCC patients should be evaluated preoperatively to improve recipient selection strategy and to 
82 develop individualized immunosuppressive and anti-cancer regiments. Microvascular invasion 83 (MVI) is widely recognized as a powerful adverse predictor for HCC recurrence after LT (Victor 84 et al., 2020; Mehta et al., 2017). However, MVI is usually difficult to assay before surgery because it depends on pathological examination. Therefore, the development of a noninvasive method that can accurately identify MVI preoperatively has become a research hotspot (Xu et al., 2019; Ma et al., 2020).

Glutamate dehydrogenase (GLDH) belongs to the amino acid dehydrogenase enzyme superfamily. It catalyzes the reversible inter-conversion of glutamate to $\alpha$-ketoglutarate and ammonia using $\mathrm{NADP}(\mathrm{H})$ and/or $\mathrm{NAD}(\mathrm{H})$ as cofactors and plays an important role in nitrogen and carbon metabolism (Oliveira et al., 2012; Spanaki et al., 2017). Glutamine is an important source of metabolic energy in many cancers (Matés et al., 2019). Consequently, as the main enzyme regulating glutamate metabolism, GLDH may play a crucial role in tumor growth (Spinelli et al., 2017; Jin et al., 2015). Jin et al. (Jin et al., 2018) found that GLDH1 mediates the metabolic reprogramming of glutaminolysis and regulates lung cancer metastasis, while Yang et al. (Yang et al., 2020) demonstrated that GLDH1-mediated glutaminolysis is associated with epidermal growth factor-promoted glioblastoma cell proliferation. Moreover, a clinical study (Piras-Straub et al., 2015) reported that GLDH is an independent predictor for HCC recurrence post-LT.

Glutamine metabolism has also been reported to be essential in angiogenesis (Huang et al., 2017). Consequently, we hypothesize that, given its role in glutamate metabolism, GLDH can serve as a serum biomarker for MVI. To date, no studies have examined the relationship between GLDH and MVI in HCC. Accordingly, we evaluated the ability of preoperative serum GLDH levels in predicting MVI and the survival of HCC patients following LT.

\section{Patients and Methods}

\section{Ethical statement}

This study was approved by the Institutional Ethics Committee for Clinical Research and Animal Trials of the First Affiliated Hospital of Sun Yat-sen University (Ethical Application Ref:2021-352). The ethical principles were in line with the Declaration of Helsinki. All patients signed an informed consent form before LT. The sole source of LTs in our study were from civilian liver donations; no organs from executed prisoners were transplanted.

\section{Patient selection}

Patients who underwent LT at the First Affiliated Hospital of Sun Yat-Sen University from January 2015 to May 2020 were recruited for our study. The inclusion criteria were: underwent LT and pathological confirmation of HCC. The exclusion criteria were: patients with a history of hepatectomy, transarterial chemoembolization, or ablation before LT; cases with incomplete medical records that made it impossible to extract all needed clinicopathological parameters; perioperative death in hospital. Distant metastases or other concurrent malignancies were considered contraindications for LT. 
122

123

124

125

126

127

128

129

130

131

132

133

134

135

136

137

138

139

140

141

142

143

144

145

146

147

148

149

150

151

152

153

154

155

156

157

158

159

160

161

162

\section{Surgical technique and immunosuppression regimens}

Generally, classic orthotopic LT was performed for the HCC patients. In patients who had renal insufficiency before surgery, piggyback LT was chosen cautiously. Venovenous bypass was not used. Our institution's immunosuppression regimen consisted of two doses of Basiliximab (20 mg each) during LT and on the fourth day post-LT. Inhibitors of calcineurin (usually Tacrolimus, in a few cases cyclosporine) and mechanistic target of rapamycin (mTOR) (usually Sirolimus) were used for maintenance immunosuppression. Depending on the side effects, the specific maintenance immunosuppression regimen was adjusted during the followup.

\section{Patient variable extraction and follow-up}

Patient variables were extracted from the electronic medical records. All serum biochemical variables were taken from the results of the last preoperative test. Tumor-related variables, including tumor size, number, and liver cirrhosis, were based on the results of the last preoperative radiological examination. MVI was diagnosed post-LT via pathological examination.

After being discharged, patients were routinely followed-up as outpatients or with phone calls. Elevated serum alpha-fetoprotein (AFP) was considered as a sign of recurrence; however, recurrence was defined based on radiological findings of recurrent lesions using contrastenhanced CT/MRI or ultrasound. Overall survival (OS) was calculated from the date of LT to the date of death or last follow-up, while recurrence-free survival (RFS) was calculated from the date of LT to the date of recurrence.

\section{Statistical analysis}

Statistical analyses were carried out using SPSS (version 23.0, IBM). Data that were not normally distributed are presented as median value and the $M$ (range), and differences between groups were detected via the Mann-Whitney $U$ test. The optimal cut-off value of GLDH was determined using receiver operating characteristic (ROC) curve analysis. To identify the risk factors and independent risk factors for MVI, univariate and multivariate logistic regression analyses were performed. Baseline variables that showed univariate significance were entered into the multivariate logistic analysis. OS and RFS were calculated using the Kaplan-Meier method and compared using the log-rank test. In all analyses, $p<0.05$ was considered statistically significant.

\section{Results}

\section{Patient characteristics}

In total, 91 patients were ultimately enrolled in our study. $92.31 \%(84)$ were male, while only $7(7.69 \%)$ were female. Most $(67,73.63 \%)$ patients were under 60 years old, while 24 (26.37\%) were over 60. Sixty-two (68.13\%) patients had AFP levels under $200 \mu \mathrm{g} / \mathrm{L}$; the remaining $29(31.87 \%$ ) had elevated AFP levels (i.e., greater than $200 \mu \mathrm{g} / \mathrm{L}$ ). The majority 
$163(90.11 \%)$ of patients were hepatitis B surface antigen-positive; accordingly, nearly $90 \%$

$164(84.62 \%)$ of the patients had hepatitis B virus-related liver cirrhosis. The radiological

165 examinations revealed that 42 patients $(46.15 \%)$ had multiple tumors, and $43(47.25 \%)$ had

166 maximum tumor diameters greater than $5 \mathrm{~cm}$. MVI was confirmed via postoperative pathological

167 examination in $29(31.87 \%)$ patients.

The following variables did not follow a normal distribution and are expressed as median value and $M$ (range): albumin/globulin: 1.20 (0.50-3), prealbumin (PA): 95 (38-365) mg/L, total bilirubin (TB): 37.30 (9-584) $\mu \mathrm{mol} / \mathrm{L}$, alanine aminotransferase (ALT): 30 (4-825) U/L, cancer antigen 125 (CA125): 61.90 (4.90-4854.80) U/mL, and GLDH: 7.30 (1.30-918.80) U/L (Table $1)$.

173

174

175

176

177

178

179

180

181

182

183

184

185

186

187

188

189

190

191

192

193

194

195

196

197

198

199

200

201

202

203

\section{Expression profiles and the optimal cut-off value of GLDH}

As illustrated in Figure 1a, preoperative serum GLDH levels were significantly higher in the MVI-positive patients $(U=454.00, p=0.000)$.

Next, we conducted a ROC curve analysis to determine the optimal cut-off value of GLDH for the presence of MVI (Fig. 1b). The optimal cut-off value of GLDH was 7.45 U/L for MVI, and the area under the curve was 0.747 (95\% confidence interval: $0.639-0.856, p=0.000)$. The sensitivity and specificity were, respectively, $79.3 \%$ and $64.5 \%$. The maximum Youden index was 0.438 .

\section{The clinical value of GLDH in predicting MVI}

GLDH levels were divided into categorical variables $(>7.45 \mathrm{U} / \mathrm{L}$ and $\leq 7.45 \mathrm{U} / \mathrm{L})$ based on the optimal cut-off value from the ROC curve. The univariate logistic regression analysis indicated that AFP $>200 \mu \mathrm{g} / \mathrm{L}(p=0.000)$, maximum diameter $>5 \mathrm{~cm}(p=0.000)$, liver cirrhosis $(p=0.008)$, and GLDH $>7.45 \mathrm{U} / \mathrm{L}(p=0.000)$ were risk factors for MVI in HCC. Furthermore, the multivariate analysis revealed that maximum diameter $>5 \mathrm{~cm}(p=0.001)$ and $\mathrm{GLDH}>7.45 \mathrm{U} / \mathrm{L}(p=0.023)$ were independent risk factors for MVI (Table 2).

\section{Prognostic significance of GLDH in the OS and RFS of HCC patients}

We produced Kaplan-Meier curves to evaluate the effect of high preoperative serum GLDH levels on the long-term survival of HCC patients following LT. As depicted in Figures $2 \mathrm{a}$ and $2 \mathrm{~b}$, patients with GLDH $>7.45 \mathrm{U} / \mathrm{L}$ had significantly poorer OS $(p=0.001)$ and RFS $(p=0.001)$ than patients with GLDH $\leq 7.45 \mathrm{U} / \mathrm{L}$. Similarly, as a powerful predictor, the predictive ability of MVI has also been verified in our cohort. Those patients with MVI were associated with worse survival (Fig. 2c, 2d).

\section{Discussion}

This study found that elevated preoperative serum GLDH levels is an effective predictor for MVI in HCC and is associated with poor prognosis post-LT. The ROC curve indicated that the optimal cut-off value of GLDH for MVI was $7.45 \mathrm{U} / \mathrm{L}$, with a sensitivity and specificity of 
204

205

206

207

$79.3 \%$ and $64.5 \%$, respectively. The multivariate analysis found that GLDH $>7.45 \mathrm{U} / \mathrm{L}$ and maximum diameter $>5 \mathrm{~cm}$ were independent risk factors for MVI. Moreover, Kaplan-Meier curves revealed that higher preoperative levels of GLDH was associated with significantly poorer OS and RFS post-LT in HCC patients.

Recurrence has become the greatest adverse factor in decreasing the survival of HCC patients after LT. Thus, effectively predicting the risk of tumor recurrence before surgery so that corresponding treatments can be carried out is vital. Since MVI is widely recognized as a powerful risk factor for HCC recurrence, the prediction of MVI may be used as a proxy of recurrence risk. Previous studies (Lee et al., 2017; Yang et al., 2019) have reported some progress in predicting MVI based on radiological evidence. Additionally, serum biomarkers such as the neutrophil-to-lymphocyte ratio or circulating tumor cells have demonstrated some promise in this area (Nitta et al., 2019; Zhou et al., 2020).

Our results demonstrate the potential value of GLDH in predicting MVI from the clinical perspective. We hypothesize that this predictive ability may be due to its critical role in glutamine metabolism. GLDH catalyzes both the oxidative deamination of glutamate to $\alpha$ ketoglutarate as well as the reductive amination in the reverse direction (Hohnholt et al., 2018). In addition to being an energy substrate for tumor metabolism, glutamine is essential in angiogenesis. Kim et al. (Kim et al., 2017) found that glutamine serves as a nitrogen source in the generation of biomass in endothelial cell proliferation. Furthermore, Huang et al. (Huang et al., 2017) reported that glutamine metabolism is essential for vessel sprouting in vitro and in vivo. Therefore, as a key enzyme in glutamine metabolism, GLDH likely regulates angiogenesis. Nevertheless, to the best of our knowledge, no studies have, to date, directly confirmed the relationship between GLDH and tumor-angiogenesis-related physiological processes. Basic research may be needed to elucidate the potential mechanisms underlying the regulation of angiogenesis by GLDH. As well, our results confirmed the effectiveness of maximum tumor diameter in predicting MVI, which is consistent with a previous study (Yan et al., 2020).

The cut-off value of GLDH predicting MVI in our study was found to be $7.45 \mathrm{U} / \mathrm{L}$, very close to the upper limit of the normal level $(7.5 \mathrm{U} / \mathrm{L})$ in our center. The possible reasons are listed as followings: Firstly, for a specific indicator, the reference ranges of the normal level in different centers are common to be different, mainly owing to different detecting methods. Secondly, the clinical significance of our study lies in indicating the existence of MVI in HCC through the increasing trend in GLDH. In contrast, no consensus exists on the specific cut-off value of GLDH, mainly due to the small sample size of our study. Therefore, studies with large samples are necessary to explore and verify the optimal cut-off value of the GLDH in future. In addition, the population characteristics of patients in each region are also different, which may introduce some individual differences into the statistical analysis.

Our results may be effective in stratifying recurrence risk in HCC patients pre-LT and may provide a reference in designing immunosuppression and anti-tumor regimens. Patients with significantly elevated preoperative serum GLDH levels may need closer monitoring after LT.

The major limitation of this study is the small sample size as mentioned above. To ensure the accuracy of the pathological diagnoses, only cases that did not undergo anti-tumor therapy 
245

246

before LT were enrolled in our study. Transarterial chemoembolization/ablation may result in the necrosis of HCC lesions, which creates difficulties in identifying MVI under the microscope. Thus, larger sample sizes will be needed in future studies.

In conclusion, our study identified the potential clinical value of GLDH in predicting MVI and the long-term prognosis of HCC patients after LT.

\section{References:}

Bodzin, A.S., Lunsford, K.E., Markovic, D., Harlander-Locke, M.P., Busuttil, R.W., and Agopian, V.G. 2017. Predicting Mortality in Patients Developing Recurrent Hepatocellular Carcinoma After Liver Transplantation: Impact of Treatment Modality and Recurrence Characteristics. ANNALS OF SURGERY 266:118-125. 10.1097/SLA.0000000000001894 Chen, W., Zheng, R., Baade, P.D., Zhang, S., Zeng, H., Bray, F., Jemal, A., Yu, X.Q., and He, J. 2016. Cancer statistics in China, 2015. CA Cancer J Clin 66:115-132. 10.3322/caac.21338 Felden, J., and Villanueva, A. 2020. Role of Molecular Biomarkers in Liver Transplantation for Hepatocellular Carcinoma. LIVER TRANSPLANTATION 26:823-831. 10.1002/1t.25731 Hohnholt, M.C., Andersen, V.H., Andersen, J.V., Christensen, S.K., Karaca, M., Maechler, P., and Waagepetersen, H.S. 2018. Glutamate dehydrogenase is essential to sustain neuronal oxidative energy metabolism during stimulation. Journal of Cerebral Blood Flow \& Metabolism 38:1754-1768. 10.1177/0271678X17714680

Huang, H., Vandekeere, S., Kalucka, J., Bierhansl, L., Zecchin, A., Brüning, U., Visnagri, A., Yuldasheva, N., Goveia, J., Cruys, B., Brepoels, K., Wyns, S., Rayport, S., Ghesquière, B., Vinckier, S., Schoonjans, L., Cubbon, R., Dewerchin, M., Eelen, G., and Carmeliet, P. 2017. Role of glutamine and interlinked asparagine metabolism in vessel formation. The EMBO journal 36:2334-2352. 10.15252/embj.201695518 Jin, L., Chun, J., Pan, C., Kumar, A., Zhang, G., Ha, Y., Li, D., Alesi, G.N., Kang, Y., Zhou, L., Yu, W., Magliocca, K.R., Khuri, F.R., Qu, C., Metallo, C., Owonikoko, T.K., and Kang, S. 2018. The PLAG1-GDH1 Axis Promotes Anoikis Resistance and Tumor Metastasis through CamKK2AMPK Signaling in LKB1-Deficient Lung Cancer. MOLECULAR CELL 69:87-99. 10.1016/j.molcel.2017.11.025

Jin, L., Li, D., Alesi, G.N., Fan, J., Kang, H., Lu, Z., Boggon, T.J., Jin, P., Yi, H., Wright, E.R., Duong, D., Seyfried, N.T., Egnatchik, R., DeBerardinis, R.J., Magliocca, K.R., He, C., Arellano, M.L., Khoury, H.J., Shin, D.M., Khuri, F.R., and Kang, S. 2015. Glutamate Dehydrogenase 1 Signals through Antioxidant Glutathione Peroxidase 1 to Regulate Redox Homeostasis and Tumor Growth. CANCER CELL 27:257-270. 10.1016/j.ccell.2014.12.006

Kim, B., Li, J., Jang, C., and Arany, Z. 2017. Glutamine fuels proliferation but not migration of endothelial cells. The EMBO journal 36:2321-2333. 10.15252/embj.201796436 Lee, S., Kim, S.H., Lee, J.E., Sinn, D.H., and Park, C.K. 2017. Preoperative gadoxetic acidenhanced MRI for predicting microvascular invasion in patients with single hepatocellular carcinoma. JOURNAL OF HEPATOLOGY 67:526-534. 10.1016/j.jhep.2017.04.024 
286

287

288

289

290

291

292

293

294

295

296

297

298

299

300

301

302

303

304

305

306

307

308

309

310

311

312

313

314

315

316

317

318

319

320

321

322

323

324

325

326

Ma, X., Tang, W., Yang, M., Xie, S., Wu, M., Lin, G., and Lu, R. 2020. Serum STIP1, a Novel Indicator for Microvascular Invasion, Predicts Outcomes and Treatment Response in Hepatocellular Carcinoma. Frontiers in Oncology 10:511. 10.3389/fonc.2020.00511 Matés, J.M., Campos-Sandoval, J.A., Santos-Jiménez, J.D.L., and Márquez, J. 2019. Dysregulation of glutaminase and glutamine synthetase in cancer. CANCER LETTERS 467:2939. 10.1016/j.canlet.2019.09.011

Mehta, N., Heimbach, J., Harnois, D.M., Sapisochin, G., Dodge, J.L., Lee, D., Burns, J.M., Sanchez, W., Greig, P.D., Grant, D.R., Roberts, J.P., and Yao, F.Y. 2017. Validation of a Risk Estimation of Tumor Recurrence After Transplant (RETREAT) Score for Hepatocellular Carcinoma Recurrence After Liver Transplant. JAMA Oncology 3:493-500. 10.1001/jamaoncol.2016.5116

Nitta, H., Allard, M., Sebagh, M., Karam, V., Ciacio, O., Pittau, G., Vibert, E., Sa Cunha, A., Cherqui, D., Castaing, D., Bismuth, H., Guettier, C., Samuel, D., Baba, H., and Adam, R. 2019. Predictive model for microvascular invasion of hepatocellular carcinoma among candidates for either hepatic resection or liver transplantation. SURGERY 165:1168-1175.

10.1016/j.surg.2019.01.012

Oliveira, T., Panjikar, S., Carrigan, J.B., Hamza, M., Sharkey, M.A., Engel, P.C., and Khan, A.R. 2012. Crystal structure of NAD+-dependent Peptoniphilus asaccharolyticus glutamate dehydrogenase reveals determinants of cofactor specificity. JOURNAL OF STRUCTURAL BIOLOGY 177:543-552. 10.1016/j.jsb.2011.10.006

Piras-Straub, K., Khairzada, K., Gerken, G., Saner, F., Treckmann, J.R., Paul, A., Canbay, A., and Herzer, K. 2015. Glutamate Dehydrogenase and Alkaline Phosphatase as Very Early Predictors of Hepatocellular Carcinoma Recurrence after Liver Transplantation. DIGESTION 91:117-127. 10.1159/000370212

Sapisochin, G., Goldaracena, N., Astete, S., Laurence, J.M., Davidson, D., Rafael, E., Castells, L., Sandroussi, C., Bilbao, I., Dopazo, C., Grant, D.R., Lázaro, J.L., Caralt, M., Ghanekar, A., McGilvray, I.D., Lilly, L., Cattral, M.S., Selzner, M., Charco, R., and Greig, P.D. 2015. Benefit of Treating Hepatocellular Carcinoma Recurrence after Liver Transplantation and Analysis of Prognostic Factors for Survival in a Large Euro-American Series. ANNALS OF SURGICAL ONCOLOGY 22:2286-2294. 10.1245/s10434-014-4273-6

Spanaki, C., Kotzamani, D., and Plaitakis, A. 2017. Widening Spectrum of Cellular and Subcellular Expression of Human GLUD1 and GLUD2 Glutamate Dehydrogenases Suggests Novel Functions. NEUROCHEMICAL RESEARCH 42:92-107. 10.1007/s11064-016-1986-x Spinelli, J.B., Yoon, H., Ringel, A.E., Jeanfavre, S., Clish, C.B., and Haigis, M.C. 2017. Metabolic recycling of ammonia via glutamate dehydrogenase supports breast cancer biomass. SCIENCE 358:941-946. 10.1126/science.aam9305

Verna, E.C., Patel, Y.A., Aggarwal, A., Desai, A.P., Frenette, C., Pillai, A.A., Salgia, R., Seetharam, A., Sharma, P., Sherman, C., Tsoulfas, G., and Yao, F.Y. 2020. Liver transplantation for hepatocellular carcinoma: Management after the transplant. AMERICAN JOURNAL OF TRANSPLANTATION 20:333-347. 10.1111/ajt.15697

Victor, D.R., Monsour, H.J., Boktour, M., Lunsford, K., Balogh, J., Graviss, E.A., Nguyen, D.T., 
327 McFadden, R., Divatia, M.K., Heyne, K., Ankoma-Sey, V., Egwim, C., Galati, J., Duchini, A.,

328 Saharia, A., Mobley, C., Gaber, A.O., and Ghobrial, R.M. 2020. Outcomes of Liver

329 Transplantation for Hepatocellular Carcinoma Beyond the University of California San

330 Francisco Criteria: A Single-center Experience. TRANSPLANTATION 104:113-121.

331 10.1097/TP.0000000000002835

332 Xu, X., Lu, D., Ling, Q., Wei, X., Wu, J., Zhou, L., Yan, S., Wu, L., Geng, L., Ke, Q., Gao, F., 333 Tu, Z., Wang, W., Zhang, M., Shen, Y., Xie, H., Jiang, W., Wang, H., and Zheng, S. 2016. Liver 334 transplantation for hepatocellular carcinoma beyond the Milan criteria. GUT 65:1035-1041.

335 10.1136/gutjnl-2014-308513

336 Xu, X., Zhang, H., Liu, Q., Sun, S., Zhang, J., Zhu, F., Yang, G., Yan, X., Zhang, Y., and Liu, X. 337 2019. Radiomic analysis of contrast-enhanced CT predicts microvascular invasion and outcome 338 in hepatocellular carcinoma. JOURNAL OF HEPATOLOGY 70:1133-1144.

339 10.1016/j.jhep.2019.02.023

340 Yan, Y., Zhou, Q., Zhang, M., Liu, H., Lin, J., Liu, Q., Shi, B., Wen, K., Chen, R., Wang, J., 341 Mao, K., and Xiao, Z. 2020. Integrated Nomograms for Preoperative Prediction of Microvascular 342 Invasion and Lymph Node Metastasis Risk in Hepatocellular Carcinoma Patients. ANNALS OF 343 SURGICAL ONCOLOGY 27:1361-1371. 10.1245/s10434-019-08071-7

344 Yang, J.D., Hainaut, P., Gores, G.J., Amadou, A., Plymoth, A., and Roberts, L.R. 2019. A global view of hepatocellular carcinoma: trends, risk, prevention and management. Nat Rev Gastroenterol Hepatol 16:589-604. 10.1038/s41575-019-0186-y Yang, L., Gu, D., Wei, J., Yang, C., Rao, S., Wang, W., Chen, C., Ding, Y., Tian, J., and Zeng, M. 2019. A Radiomics Nomogram for Preoperative Prediction of Microvascular Invasion in Hepatocellular Carcinoma. Liver Cancer 8:373-386. 10.1159/000494099 Yang, R., Li, X., Wu, Y., Zhang, G., Liu, X., Li, Y., Bao, Y., Yang, W., and Cui, H. 2020. EGFR activates GDH1 transcription to promote glutamine metabolism through MEK/ERK/ELK1 pathway in glioblastoma. ONCOGENE 39:2975-2986. 10.1038/s41388-020-1199-2 Zhou, J., Sun, H.C., Wang, Z., Cong, W.M., Wang, J.H., Zeng, M.S., Yang, J.M., Bie, P., Liu, L.X., Wen, T.F., Han, G.H., Wang, M.Q., Liu, R.B., Lu, L.G., Ren, Z.G., Chen, M.S., Zeng, Z.C., Liang, P., Liang, C.H., Chen, M., Yan, F.H., Wang, W.P., Ji, Y., Cheng, W.W., Dai, C.L., Jia, W.D., Li, Y.M., Li, Y.X., Liang, J., Liu, T.S., Lv, G.Y., Mao, Y.L., Ren, W.X., Shi, H.C., Wang, W.T., Wang, X.Y., Xing, B.C., Xu, J.M., Yang, J.Y., Yang, Y.F., Ye, S.L., Yin, Z.Y., Zhang, B.H., Zhang, S.J., Zhou, W.P., Zhu, J.Y., Liu, R., Shi, Y.H., Xiao, Y.S., Dai, Z., Teng, G.J., Cai, J.Q., Wang, W.L., Dong, J.H., Li, Q., Shen, F., Qin, S.K., and Fan, J. 2018. Guidelines for Diagnosis and Treatment of Primary Liver Cancer in China (2017 Edition). Liver Cancer 7:235-260. 10.1159/000488035 Preoperative circulating tumor cells to predict microvascular invasion and dynamical detection indicate the prognosis of hepatocellular carcinoma. BMC CANCER 20:1047. 10.1186/s12885020-07488-8 
Figure 1

The correlation and optimal cut-off value between preoperative serum GLDH levels and MVI.

a Distribution of GLDH levels in patients with and without MVI. b ROC curve of GLDH for MVI. AUC, area under the curve; GLDH, glutamate dehydrogenase; MVI, microvascular invasion; ROC, receiver operating characteristic.

a

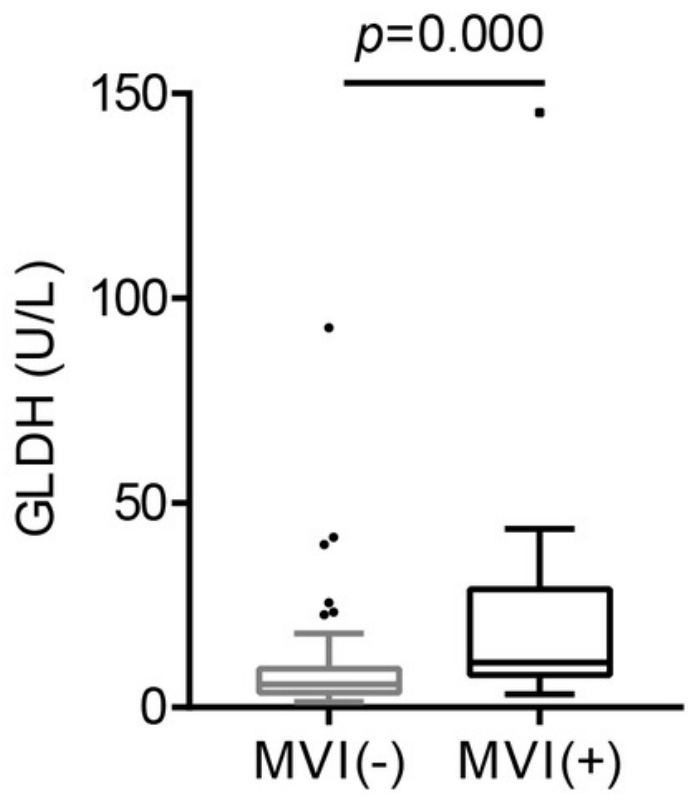

b

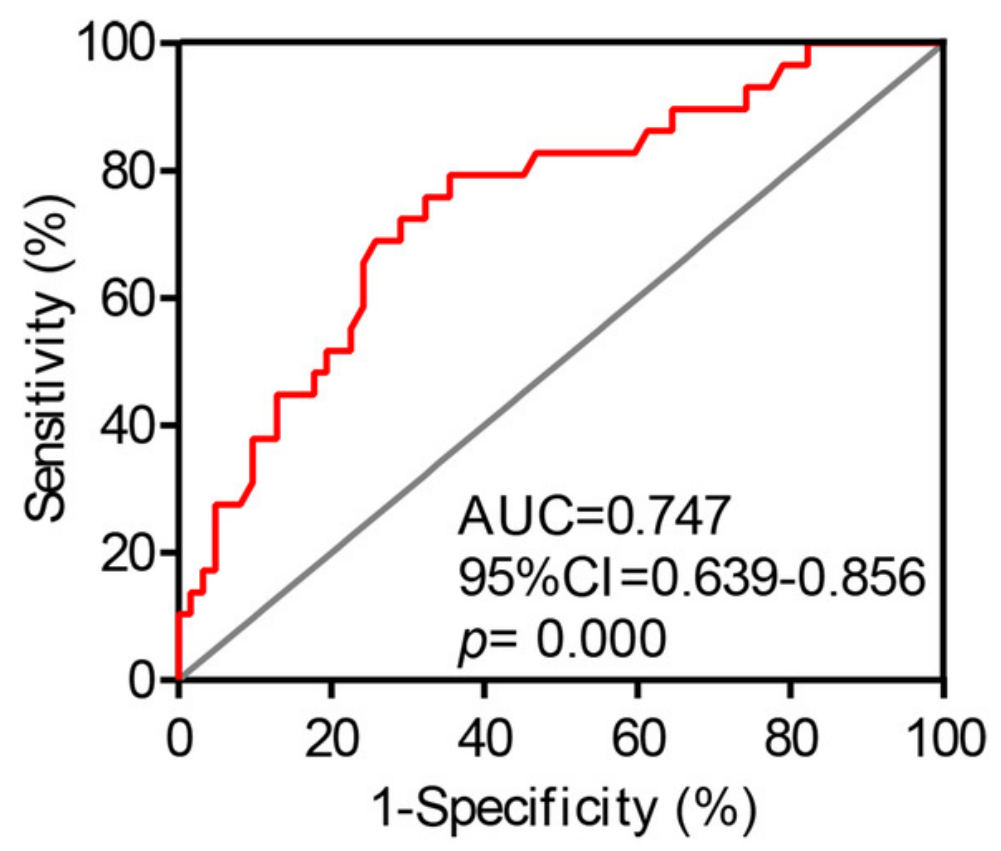


Figure 2

Kaplan-Meier analysis in patients with different levels of GLDH $(a, b)$ and patients with or without MVI (c, d). GLDH, glutamate dehydrogenase; MVI, microvascular invasion

a

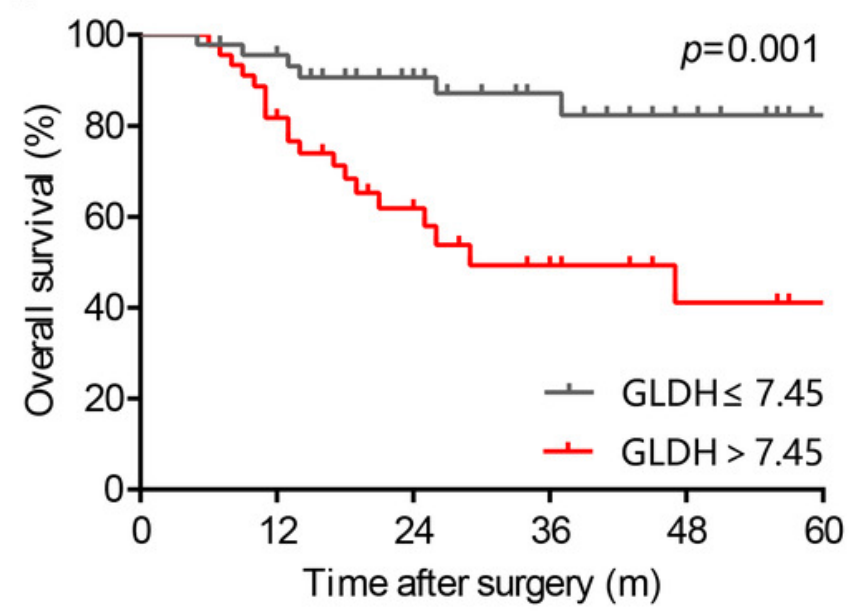

c

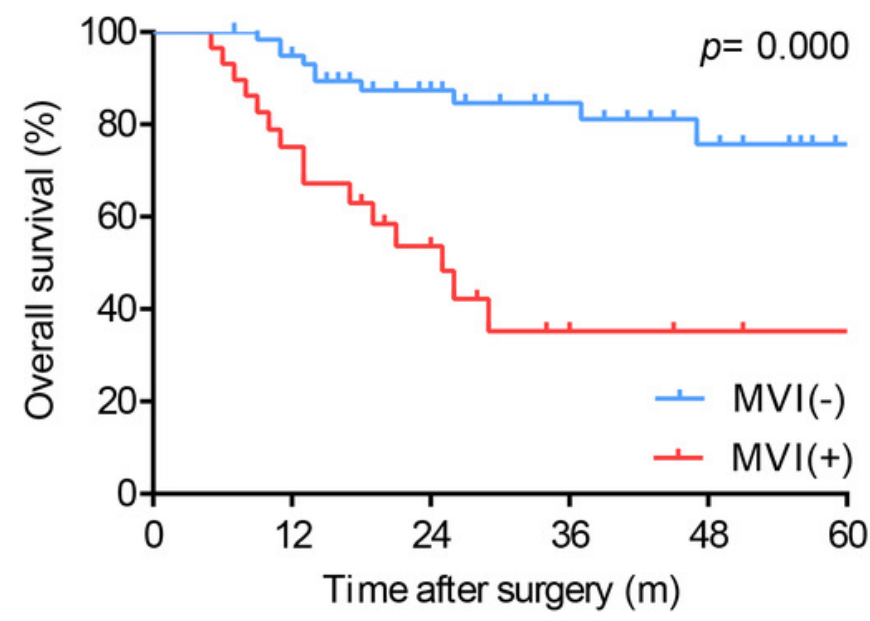

b

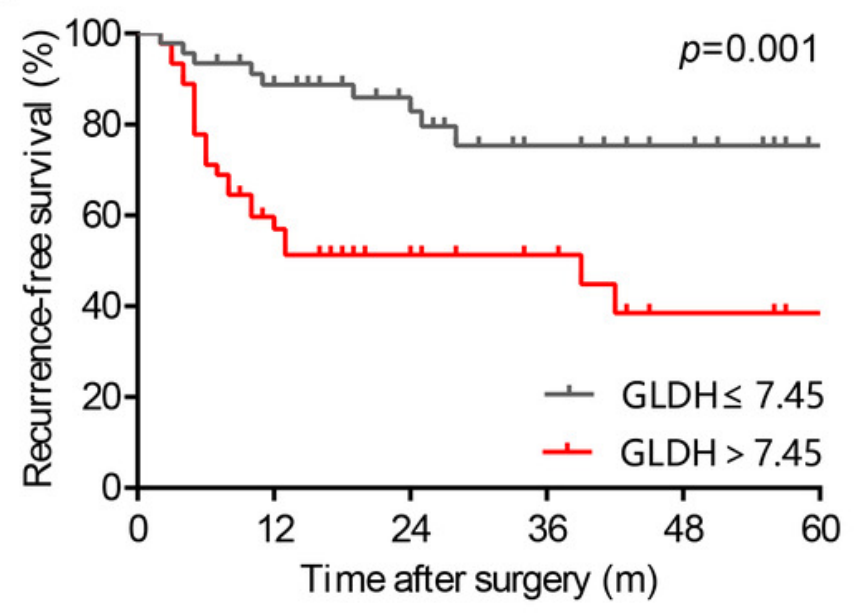

d

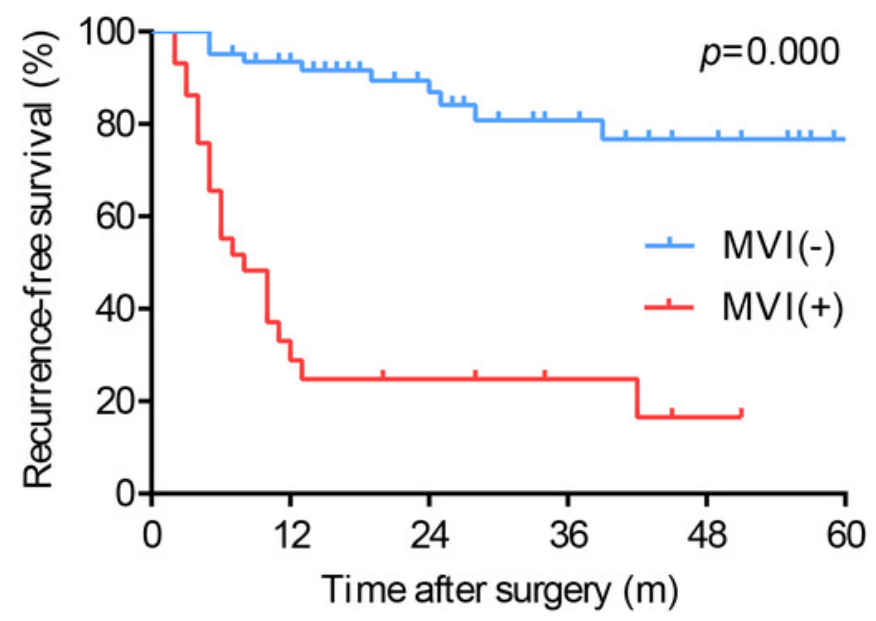




\section{Table 1 (on next page)}

Baseline characteristics of 91 patients.

AFP, alpha-fetoprotein; HBsAg, hepatitis B surface antigen; MVI, microvascular invasion; PA, prealbumin; ALT, alanine aminotransferase; GLDH, glutamate dehydrogenase. 
1 Table 1. Clinical variables of 91 patients.

\begin{tabular}{|c|c|}
\hline Variables & No. of patients $(\%) / \operatorname{Median}(M)$ \\
\hline \multicolumn{2}{|l|}{ Sex } \\
\hline Male & $84(92.31)$ \\
\hline Female & $7(7.69)$ \\
\hline \multicolumn{2}{|l|}{ Age (years) } \\
\hline$\leq 60$ & $67(73.63)$ \\
\hline$>60$ & $24(26.37)$ \\
\hline \multicolumn{2}{|l|}{$\operatorname{AFP}(\mu \mathrm{g} / \mathrm{L})$} \\
\hline$\leq 200$ & $62(68.13)$ \\
\hline$>200$ & $29(31.87)$ \\
\hline \multicolumn{2}{|l|}{ HBsAg } \\
\hline Negative & $9(9.89)$ \\
\hline Positive & $82(90.11)$ \\
\hline \multicolumn{2}{|c|}{ Maximum diameter $(\mathrm{cm})$} \\
\hline$\leq 5$ & $48(52.75)$ \\
\hline$>5$ & $43(47.25)$ \\
\hline \multicolumn{2}{|l|}{ Tumor number } \\
\hline Single & $49(53.85)$ \\
\hline Multiple & $42(46.15)$ \\
\hline \multicolumn{2}{|l|}{ MVI } \\
\hline Negative & $62(68.13)$ \\
\hline Positive & $29(31.87)$ \\
\hline \multicolumn{2}{|l|}{ Liver cirrhosis } \\
\hline Negative & $14(15.38)$ \\
\hline Positive & $77(84.62)$ \\
\hline Albumin/Globulin & $1.20(0.50-3)$ \\
\hline $\mathrm{PA}(\mathrm{mg} / \mathrm{L})$ & $95(38-365)$ \\
\hline $\mathrm{TB}(\mu \mathrm{mol} / \mathrm{L})$ & $37.30(9-584)$ \\
\hline $\operatorname{ALT}(\mathrm{U} / \mathrm{L})$ & $30(4-825)$ \\
\hline CA125 (U/mL) & $61.90(4.90-4854.80)$ \\
\hline GLDH (U/L) & $7.30(1.30-918.80)$ \\
\hline
\end{tabular}

2 AFP, alpha-fetoprotein, HBsAg, hepatitis B surface antigen, MVI, microvascular invasion, PA, prealbumin, ALT,

3 alanine aminotransferase, GLDH, glutamate dehydrogenase

4 


\section{Table 2 (on next page)}

Univariate and multivariate logistic regression analysis for the presence of MVI.

AFP, alpha-fetoprotein; HBsAg, hepatitis B surface antigen; MVI, microvascular invasion; PA, prealbumin; ALT, alanine aminotransferase; GLDH, glutamate dehydrogenase. 
1 Table2. Univariable and multivariable logistic analysis of risk factors to MVI

\begin{tabular}{|c|c|c|c|c|c|c|}
\hline \multirow[t]{2}{*}{ Variables } & \multirow[t]{2}{*}{ OR } & \multicolumn{2}{|c|}{ Univariate analysis } & \multirow[t]{2}{*}{ OR } & \multicolumn{2}{|c|}{ Multivariate analysis } \\
\hline & & $\begin{array}{l}\text { HR }(95 \% \\
\text { CI) }\end{array}$ & $p$ & & $\begin{array}{l}\text { HR }(95 \% \\
\text { CI) }\end{array}$ & \\
\hline Sex (male vs female) & & & 0.999 & & & \\
\hline Age, y $(>60$ vs $\leq 60)$ & 0.64 & $0.22-1.83$ & 0.402 & & & \\
\hline AFP, $\mu \mathrm{g} / \mathrm{L}(>200$ vs $\leq 200)$ & 5.90 & $2.24-15.59$ & 0.000 & 3.06 & $0.96-9.75$ & 0.059 \\
\hline HBsAg (positive vs negative) & 1.72 & $0.33-8.84$ & 0.517 & & & \\
\hline Maximum diameter, $\mathrm{cm}(>5 \mathrm{vs} \leq 5)$ & 15.28 & $4.65-50.19$ & 0.000 & 8.85 & $2.41-32.56$ & 0.001 \\
\hline Tumor number (multiple vs single) & 1.39 & $0.57-3.36$ & 0.467 & & & \\
\hline Liver cirrhosis (positive vs negative) & 0.20 & $0.06-0.65$ & 0.008 & 0.75 & $0.19-3.02$ & 0.687 \\
\hline Albumin/Globulin & 0.52 & $0.17-1.65$ & 0.267 & & & \\
\hline $\mathrm{PA}(\mathrm{mg} / \mathrm{L})$ & 1.01 & $1.00-1.01$ & 0.185 & & & \\
\hline $\mathrm{TB}(\mu \mathrm{mol} / \mathrm{L})$ & 0.99 & $0.98-1.00$ & 0.122 & & & \\
\hline $\operatorname{ALT}(\mathrm{U} / \mathrm{L})$ & 1.00 & $1.00-1.01$ & 0.248 & & & \\
\hline CA125 (U/mL) & 1.00 & $1.00-1.00$ & 0.343 & & & \\
\hline GLDH, U/L $(>7.45$ vs $\leq 7.45)$ & 6.97 & $2.47-19.68$ & 0.000 & 4.01 & $1.21-13.34$ & 0.023 \\
\hline
\end{tabular}

2 AFP, alpha-fetoprotein, HBsAg, hepatitis B surface antigen, MVI, microvascular invasion, PA, prealbumin, ALT, 3 alanine aminotransferase, GLDH, glutamate dehydrogenase 